\title{
Olfactory Dysfunction Correlates with Amyloid- $\beta$ Burden in an Alzheimer's Disease Mouse Model
}

\author{
Daniel W. Wesson, ${ }^{1,3}$ Efrat Levy, ${ }^{2,4,5}$ Ralph A. Nixon, ${ }^{2,5,6}$ and Donald A. Wilson ${ }^{1,3}$ \\ ${ }^{1}$ Emotional Brain Institute and ${ }^{2}$ Center for Dementia Research, Nathan S. Kline Institute for Psychiatric Research, Orangeburg, New York 10962, and \\ Departments of ${ }^{3}$ Child and Adolescent Psychiatry, ${ }^{4}$ Pharmacology, ${ }^{5}$ Psychiatry, and ${ }^{6}$ Cell Biology, New York University School of Medicine, New York, \\ New York 10016
}

\begin{abstract}
Alzheimer's disease often results in impaired olfactory perceptual acuity-a potential biomarker of the disorder. However, the usefulness of olfactory screens to serve as informative indicators of Alzheimer's is precluded by a lack of knowledge regarding why the disease impacts olfaction. We addressed this question by assaying olfactory perception and amyloid- $\beta(\mathrm{A} \beta)$ deposition throughout the olfactory system in mice that overexpress a mutated form of the human amyloid- $\beta$ precursor protein. Such mice displayed progressive olfactory deficits that mimic those observed clinically-some evident at 3 months of age. Also, at 3 months of age, we observed nonfibrillar A $\beta$ deposition within the olfactory bulb-earlier than deposition within any other brain region. There was also a correlation between olfactory deficits and the spatial-temporal pattern of $\mathrm{A} \beta$ deposition. Therefore, nonfibrillar, versus fibrillar, $\mathrm{A} \beta$-related mechanisms likely contribute to early olfactory perceptual loss in Alzheimer's disease. Furthermore, these results present the odor cross-habituation test as a powerful behavioral assay, which reflects $\mathrm{A} \beta$ deposition and thus may serve to monitor the efficacy of therapies aimed at reducing $\mathrm{A} \beta$.
\end{abstract}

\section{Introduction}

Alzheimer's disease (AD) is a progressively debilitating brain disease wherein patients suffer from sensory, motor, and cognitive loss. Currently, much interest exists in establishing methods to diagnose $\mathrm{AD}$ before the irreversible deterioration of the brain characteristic of the disease. One potential avenue in the early diagnosis of $\mathrm{AD}$ is the use of olfactory sensory dysfunction in combination with neuropsychological measures (Murphy, 1999; Albers et al., 2006). Persons displaying AD often have a reduced ability to detect, discriminate, and identify odors (for review, see Mesholam et al., 1998; Murphy, 1999). However, the usefulness of olfactory screens to serve as an informative biomarker for $\mathrm{AD}$ is precluded by a lack of knowledge regarding why $\mathrm{AD}$ impacts olfaction. Understanding the relationship between AD neuropathological progression and the occurrence of atypical olfactory perception will be an important step forward in this quest.

$\mathrm{AD}$ is pathologically defined by the presence of amyloid- $\beta$ $(\mathrm{A} \beta$ ) plaques and neurofibrillary tangles (NFTs) within the brain. Plaques are mostly composed of $A \beta$, which derives from amyloid- $\beta$ precursor protein (APP). NFTs are made of hyperphosphorylated forms of the microtubule-associated protein tau.

Received Sept. 17, 2009; revised Nov. 4, 2009; accepted Nov. 5, 2009.

This work was supported by National Institutes of Health Grants DC003906 (D.A.W.) and AG017617 (R.A.N.) and Alzheimer's Association Grant IIRG0759699 (E.L.). We thank Drs. Gurjinder Kaur for advice with amyloid measurements, Monika Pawlik for assistance with mice breeding and genotyping, and Dun-Sheng Yang for thoughtful discussions regarding this project. D.W.W. and D.A.W. conceived of this project. E.L. and R.A.N. contributed valuable advice. D.W.W. collected and analyzed data. All authors discussed the results and their implications and contributed to manuscript preparation.

Correspondence should be addressed to Daniel W. Wesson, Nathan S. Kline Institute for Psychiatric Research, Orangeburg, NY 10962. E-mail: dwesson@nki.rfmh.org.

DOI:10.1523/JNEUROSCI.4622-09.2010

Copyright $\odot 2010$ the authors $\quad 0270-6474 / 10 / 300505-10 \$ 15.00 / 0$
Together, the presence of these markers represents the classic diagnostic hallmarks of AD (Alzheimer et al., 1995). Models of $\mathrm{AD}$-related tauopathy and amyloidosis are well established as contributing to our understanding of the physiological and cognitive aspects of the disease (Lee et al., 2001; Hardy and Selkoe, 2002). Currently, however, neither model has been directly tested in relation to understanding the cause of olfactory dysfunction in AD.

The mechanisms of AD-related olfactory sensory loss can be uniquely explored through the use of APP transgenic mouse models (Van Dijck et al., 2008; Zhuo et al., 2008; Guérin et al., 2009; Montgomery et al., 2009; Young et al., 2009). The neural mechanisms of odor processing in rodents are relatively understood. Olfaction involves processing stages spanning from sensory neuron input to the olfactory bulb, decoding and plasticity in the piriform cortex, and ultimately downstream neurons in the hippocampus (Wachowiak and Shipley, 2006; Wilson and Stevenson, 2006). Although studies exist showing olfactorybehavior deficits in APP transgenic mice (odor reversal learning, odor memory, etc.) (Van Dijck et al., 2008; Zhuo et al., 2008; Guérin et al., 2009; Montgomery et al., 2009; Young et al., 2009), no studies are available directly relating $\mathrm{A} \beta$ in the olfactory system with olfactory perceptual loss. The extent of $\mathrm{A} \beta$ pathology across this system and the involvement of these regions in ADrelated olfactory sensory deficits remains mostly unexplored (but see Price et al., 1991; Kovács, 2004). Furthermore, despite the fundamental role of $\mathrm{A} \beta$ in modulating neural processing (Selkoe, 2008), whether $A \beta$ plays a role in contributing to $A D$-related atypical olfactory behaviors is unknown.

Here, we addressed the relationship between $A \beta$ deposition within olfactory processing networks and odor perception in the 
Tg2576 AD mouse model (Hsiao et al., 1996) at sequential life stages. We found evidence to suggest that olfactory deficits in AD may stem from deposition of nonfibrillar $\mathrm{A} \beta$ within the first synaptic processing stage of the olfactory system.

\section{Materials and Methods}

Mice. Mice bred and maintained within the Nathan S. Kline Institute for Psychiatric Research animal facility were used. Tg2576 mice were generated previously by overexpressing the 695 aa isoform of human APP containing the KM670/671NL mutation, as described previously (Hsiao et al., 1996). Age-matched nontransgenic [wild-type (WT)] mice on B6SJLF1/J background were used as controls. To examine the possible contributions of accumulating $\mathrm{A} \beta$ pathology throughout life on olfaction, we used four separate age groups: 3 - to 4-month-old (Tg, $n=5,5$ males; and WT, $n=6$, 2 females, 4 males), 6- to 7-month-old (Tg, $n=5$, 3 females, 2 males; and WT, $n=4,2$ females, 2 males), 16-month-old (Tg, $n=6,6$ males; and WT, $n=9,9$ males), and 21- to 29-month-old mice (mean \pm SEM, $23.5 \pm 0.6$; Tg, $n=6$, 2 females, 4 males; and WT, $n=8,4$ females, 4 males). Mice were genotyped by PCR analysis of tail DNA using standard methods. All experiments were conducted in accordance with the guidelines of the National Institutes of Health and were approved by the Institutional Animal Care and Use Committee of the Nathan S. Kline Institute.

Olfactory behavior test. Mice were screened for olfactory deficits using an odor cross-habituation test (see Fig. 1a) (Sundberg et al., 1982; Wilson and Linster, 2008). Odors ( $n=7$; limonene, ethyl valerate, isoamyl acetate, pentanol, heptanone, propyl butyrate, and nonane; Sigma-Aldrich) were diluted $1 \times 10^{-3}$ in mineral oil and applied to a cotton applicator stick, which was then enclosed in a piece of odorless plastic tubing to prevent contact of the liquid odor with the testing chamber or animal yet still allow volatile odor delivery. Notably, such an odor presentation method controls for the influence of visual and/or somatosensory influences on odor investigation. Odors were delivered for four successive trials (one block), $20 \mathrm{~s}$ each, separated by $30 \mathrm{~s}$ intertrial intervals, by inserting the odor stick into a port on the side of the animal's home cage (see Fig. 1a). Home cage testing was chosen over testing in a separate apparatus to minimize potential influences of stress and anxiety (because of the new environment/context) on the behavioral measures. Testing took place during the light phase of the animals' $12 \mathrm{~h}$ dark/light cycle, over two daily sessions (three to four odors/session) separated by $24-48$ h. The duration of time spent investigating, defined as snout-oriented sniffing within $1 \mathrm{~cm}$ of the odor presentation port, was recorded across all trials by a single observer blind to genotypes (D. W. Wesson). Home cages were cleaned with fresh corn cob bedding $24-48 \mathrm{~h}$ before behavioral testing to reduce unnecessary background odors, yet still allow for adaptation to the new bedding. The stainless-steel food bin and water bottle were removed from cages immediately before testing.

Histology. Tissue collection was performed after either urethane anesthesia $(1 \mathrm{mg} / \mathrm{kg}$, i.p.) and transcardial perfusion with $10 \%$ formalin (3- to 4-, 6- to 7-, and 21- to 29-month-old mice) or $\mathrm{CO}_{2}$ overexposure (16month-old mice; experimental constraints prevented the use of a single killing agent for all groups). Mice were decapitated, and brains were rapidly removed over ice. Hemibrains from 3- to 4-, 6- to 7-, and 21- to 29 -month-old mice were placed in $10 \%$ formalin for fixation. Hemibrains from each animal were coronally sectioned $(40 \mu \mathrm{m})$ on a microtome. A subset of these sections were slide-mounted and immersed in a solution of filtered $1 \%$ Thioflavin-S for $10 \mathrm{~min}$ (Sigma-Aldrich), rinsed (three times) for $1 \mathrm{~min}$ in $\mathrm{dd}_{2} \mathrm{O}$, and subsequently coverslipped with Vectashield hardmount with $4^{\prime}$,6-diamidino-2-phenylindole (DAPI) (Vector Laboratories) for nuclear counterstain. The remaining sections were left floating in Tris-buffered saline (TBS) for 4G8 (anti-A $\beta$ ) immunohistochemistry (Mi et al., 2007). Sections were washed (three times at $5 \mathrm{~min}$ ) in TBS after which they were treated with $85 \%$ formic acid for 5 min to enhance amyloid staining. Sections were then washed in blocking buffer $(0.05 \mathrm{M}$ Tris-HCl, pH 7.6, $0.9 \% \mathrm{NaCl}, 0.25 \%$ Triton X-100, 20\% normal goat serum, and $0.2 \%$ bovine serum albumin) three times (10 min each) before incubating for $12 \mathrm{~h}$ in $4 \mathrm{G} 8$ primary antibody at $4{ }^{\circ} \mathrm{C}$ (Signet Laboratories; 1:200 in blocking buffer). Sections were rinsed (three times at $5 \mathrm{~min}$ ) in blocking buffer before incubating for $2 \mathrm{~h}$ at room temperature in Alexa 488 secondary antibody (Invitrogen). Finally, sections were rinsed in TBS (three times at $5 \mathrm{~min}$ ), placed on slides, and coverslipped with Vectashield hardmount with DAPI (Vector). Brains from all age groups were stained during the same procedure and a positive control section (containing the hippocampus from a 16-monthold Tg mouse) was placed on all slides of young (3- to 4- and 6- to 7-month-old) mice. Optical scans of brain sections were collected within $7 \mathrm{~d}$ after staining by use of a Zeiss Axioscope microscope (model 200M) and a Zeiss digital camera (Carl Zeiss). Sections were excited at $488 \mathrm{~nm}$, and, because of the similar, yet still quantifiably different emission spectra of Thioflavin-S/Alexa 488 and DAPI, emission from all fluorophores was captured with a FITC filter.

Data analysis. For analysis of olfactory behavior data, all raw investigatory values (in seconds) were pooled within animals and organized according to odor presentation (trial) number. Because separate groups of mice were used within each age group, two-way ANOVAs versus repeated-measured ANOVAs were used to examine the influence of age on behavior. First, as a measure of novel odor investigation, the durations of all trial 1 odor investigations were pooled within each genotype and age group and analyzed using two-way ANOVAs for independent groups (eight) followed by post hoc group comparisons using Fisher's PLSD. Second, as a measure of odor habituation, the raw investigatory values were normalized to the maximum investigatory duration per animal for each odor (max during trials $1-4$ ). The maximum investigation duration was assigned a value of 1 , and the lesser investigation times a proportion of 1 . These normalized investigation probabilities of subjects for each odor were analyzed using two-way ANOVAs for independent groups (eight) and repeated measures (four trials) within each group. Normalized odor investigation durations within individual trials were collapsed across all odors within groups across and analyzed using one-way ANOVAs for independent groups (eight). Post hoc group comparisons after a significant ANOVA result were made using Fisher's PLSD tests. Finally, normalized investigation data were again used to determine effects of genotype on odor cross-habituation (discrimination). To calculate this "cross-habituation index" (Rankin et al., 2009), the normalized investigatory values from all fourth trial odor presentations were subtracted from the following first trial odor presentations. These crosshabituation values were then analyzed using one-way ANOVAs for independent groups (four) followed by post hoc group comparisons using Fisher's PLSD.

Histological analysis of $\mathrm{A} \beta$ levels was performed in NIH ImageJ (http://rsbweb.nih.gov/ij). Six individual brain areas, the olfactory bulb (OB), orbitofrontal cortex (OFC), anterior and posterior piriform cortices (aPCX and pPCX), dorsal hippocampus (Hipp), and lateral entorhinal cortex (Ent) were analyzed for levels of Thioflavin-S and anti-A $\beta$ staining to quantify $\mathrm{A} \beta$ deposition (percentage area) across olfactory structures. Fluorescence levels of anti-A $\beta$ 4G8 and Thioflavin-S were thresholded, and regions of interest (ROIs) were determined with the guidance of the DAPI counterstain and standard anatomical coordinates (Paxinos and Franklin, 2000).

$\mathrm{A} \beta$ deposition area (percentage) was quantified within each ROI separately. For measurements of $A \beta$ deposition within the $O B$, individual layers were manually outlined using DAPI-labeled anatomical layers as guides (see Fig. $2 a$ ). The aPCX and pPCX were separated by the disappearance of the lateral olfactory tract (LOT) (see Fig. $3 a$, dashed rectangle). Individual cell layers within the PCX were manually outlined using DAPI labeling as a guide. To quantify $\mathrm{A} \beta$ deposition in the OFC, we selected a rectangular area within the medial OFC (see Fig. $4 a$, dashed region). We selected a diagonal rectangular area within the Ent to quantify $\mathrm{A} \beta$ deposition (see Fig. $4 b$, dashed region). To quantify $\mathrm{A} \beta$ deposition in the Hipp, we manually outlined all Hipp regions (dentate, CA1, $\mathrm{CA} 2$, and CA3) (see Fig. $4 c$, dashed region).

$\mathrm{A} \beta$ deposition (percentage area) (Mi et al., 2007) was defined as the cumulative area of fluorescent pixels above threshold within each ROI. At least three coronal brain sections (range, three to four) containing each ROI per mouse were used for analysis. Percentage area values were in some cases analyzed using one-way ANOVAs for independent groups followed by post hoc group comparisons using Fisher's PLSD. Percentage 
a

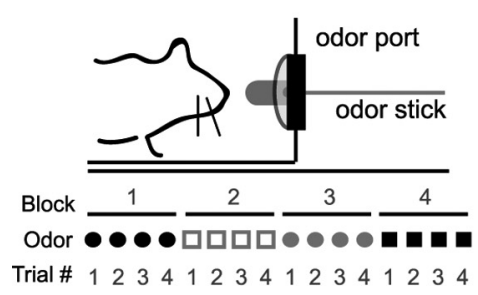

C

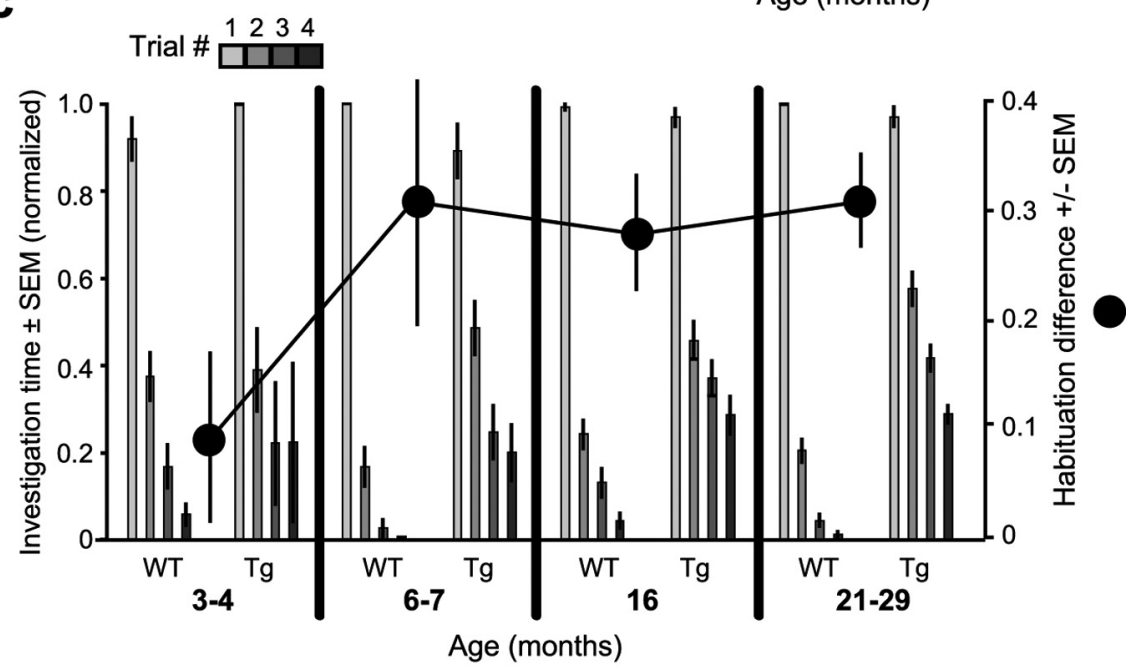

d

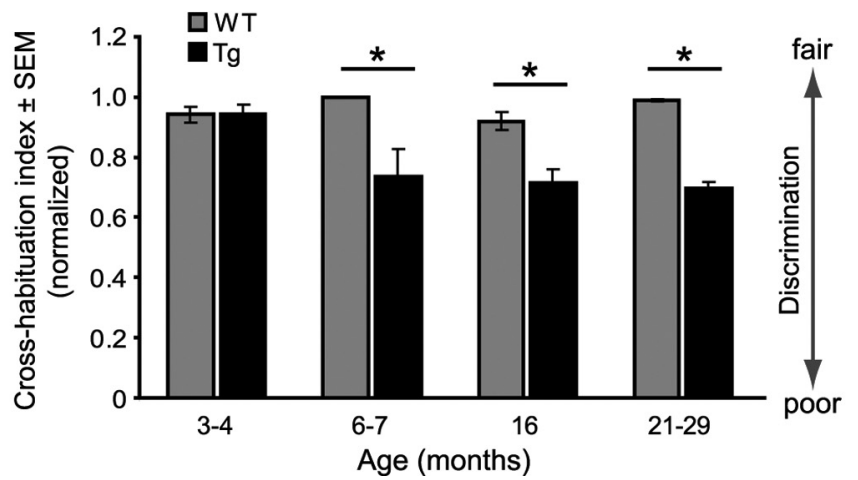

Figure 1. Progressive and age-dependent emergence of AD-like atypical olfactory behavior in APP transgenic mice. Results from an odor cross-habituation test from 3- to 4-, 6- to 7-, 16-, and 21- to 29-month-old Tg2576 (Tg) and age-matched control mice (WT) (for sample sizes, see Materials and Methods). $\boldsymbol{a}$, 0dor cross-habituation task design. Odors were presented by saturating an "odor stick" with odor, placing this inside a hollow plastic tube, and inserting this into a stainless-steel "odor port." Odors were presented in blocks of four successive trials (see Materials and Methods). $\boldsymbol{b}$, Investigation times for all trial 1 odor presentations. ${ }^{*} p<0.0001$, Tg versus WT. c, Odor habituation (normalized) (see Materials and Methods) across four successive odor presentation trials (bars). Habituation difference (dots) indicate the difference between the genotypes (WT vs Tg) within each age group in their percentage habituation across all four trials (see Materials and Methods). $\boldsymbol{d}$, Cross-habituation indices (discrimination) of Tg and WT mice. Cross-habituation index, The difference between the duration of the previous trial 4 odor investigation compared with that of the following trial 1 presentation. ${ }^{*} p<0.0001$, Tg versus WT. All statistics are ANOVA followed by Fisher's PLSD. Error bars indicate SEM.

area values for each ROI within each section were treated as independent measures for analysis.

All statistical analyses were performed in StatVIEW (SAS Institute) or in MatLab (Mathworks). All values are reported as mean \pm SEM unless otherwise stated.

\section{Results}

Our primary goal was to establish whether there is a relationship between $\mathrm{A} \beta$ neuropathology and olfactory perception in the
Tg2576 APP transgenic mouse model (Hsiao et al., 1996). We began to address this by examining odor-guided behavior in an odor cross-habituation task, which allows for measurements of novel odor investigation, odor learning and memory (habituation), and odor discrimination (i.e., cross-habituation) - all within a single behavioral test. In this test, mice were presented in their home cages with cotton applicator sticks laced with odor (see Materials and Methods) (Fig. 1a). Each odor was delivered across four successive trials (20 s duration, 30 s intertrial interval), and the duration of time spent investigating the odor was recorded across trials allowing quantification of novel odor investigation, odor habituation, and odor crosshabituation (discrimination).

Habituation (and the generalization thereof, tested as cross-habituation) is a form of implicit or nondeclarative memory and is not hippocampus dependent. One of the advantages of using odor habituation and cross-habituation as an assay in the present studies is that the synaptic mechanisms of short-term habituation (as tested here) are known. Shortterm odor habituation is attributable to a metabotropic glutamate receptor-mediated, homosynaptic depression of afferent synapses from the olfactory bulb in piriform cortex (Wilson and Linster, 2008). The odor specificity of this cortical adaptation is likely attributable to plasticity of intracortical association fiber synaptic plasticity within piriform cortex (Linster et al., 2009). Thus, although not a long-term memory task, odor habituation and its specificity allow assays of two very specific forms of synaptic plasticity and local circuit function that may be affected by ADrelated pathology.

\section{Atypical novel odor investigation behavior in Tg2576 mice}

We began our investigation into the possible olfactory deficits expressed by $\mathrm{AD}$ model mice by examining odor investigation behaviors in each age group of Tg2576 mice. The time spent investigating novel odors can be an indicator of arousal/motivation (Baum and Keverne, 2002) or habituation (Wilson and Linster, 2008). We pooled all novel (trial 1) odor investigation durations across all odors $(n=7)$ and animals within each age group and genotype. As shown in Figure $1 b$, we found that 16 month and older (21-29 months) APP transgenic (Tg) mice investigated trial 1 odors longer than age-matched controls (WT). ANOVA on all investigation times revealed a significant effect of genotype $\left(F_{(1,272)}=66.4 ; p<0.0001\right)$ and age $\left(F_{(3,270)}=10.5 ; p<0.0001\right)$. In particular, both $16-\left(F_{(1,87)}=\right.$ 36.7; $p<0.0001)$ and 21 - to 29 -month-old Tg mice $\left(F_{(1,95)}=\right.$ 
88.4; $p<0.0001)$ investigated trial 1 odors significantly longer compared with WT mice (Fig. 1b). To assess whether this tendency for aged Tg mice to investigate trial 1 odors for prolonged periods of time reflected simple hyperactivity and thus enhanced numbers of approaches to the odor port, in addition to total investigation duration, we also analyzed the number of sniff bouts. We found that 16- and 21- to 29-month-old Tg mice displayed statistically similar numbers of sniff bouts in response to trial 1 odors [mean \pm SEM, 16 months: $(\mathrm{Tg}) 1.6 \pm 0.2$, (WT) $1.6 \pm 0.1 ; 21-29$ months: (Tg) $1.8 \pm 0.2$, (WT) $1.5 \pm 0.2$; twoway ANOVA, $p>0.05$, Tg vs WT within each age], suggesting that the elevated odor investigation behavior displayed in 16- and 21- to 29-month-old APP Tg mice reflects, at least within the context of this task, deficient short-term odor habituation.

\section{Tg2576 mice fail to normally habituate to repeated odor exposure}

Previous behavioral and physiological work has demonstrated that habituation to repeated odor exposure on a short timescale $(\sim 30 \mathrm{~s})$ is a process mediated by group III metabotropic glutamate receptors (mGluRIII) (Best and Wilson, 2004) located on presynaptic nerve terminals in the olfactory piriform cortex (PCX) (Wada et al., 1998). Indeed, blocking mGluRIII receptors in vivo at the level of the PCX prevents cortical odor adaptation (Best and Wilson, 2004) and results in a failure to habituate normally to odor exposure (Yadon and Wilson, 2005). Since, here, we found that $\mathrm{AD}$ model mice possess different capacities to habituate to novel (trial 1) odors within a single $20 \mathrm{~s}$ exposure (Fig. $1 b$ ), we next assessed odor habituation over repeated odor exposures in each of four age groups of mice. To do this, odor investigation durations across all trials (four total; including the data from trial 1) (Fig. 1b) of the odor cross-habituation task were analyzed for differences between groups. Because of the significant differences shown in Figure $1 b$ in the investigation levels of the groups within trial 1 , all data were normalized before statistical analysis to the maximum odor investigation duration within each individual odor presentation block (trials 1-4) (see Materials and Methods).

We found that, beginning at 3-4 months of age, APP Tg mice displayed an increased latency to habituate across odor presentations compared with age-matched WT controls (Fig. 1c). Across all age groups, there was a significant overall genotype effect $\left(F_{(1,266)}=98.2 ; p<0.0001\right)$, an age effect $\left(F_{(3,266)}=4.6 ; p<\right.$ $0.005)$, and a significant interaction $\left(F_{(3,266)}=18.2 ; p<0.0001\right)$. Furthermore, within each age group, there was a significant repeated-measures effect $(p<0.0001)$. The significant effect of repeated measures indicated that all groups were eventually able to habituate to odors by the fourth trial, however, with slight differences among the groups (Fig. 1c). WT mice in the 6- to 7-, 16-, and 21- to 29-month-old age groups habituated more rapidly to odors compared with their age-matched Tg counterparts-achieving statistical significance by the second trial $[6-7$ months $\left(F_{(1,37)}=22.4 ; p<0.0001\right) ; 16$ months $\left(F_{(1,88)}=16.2\right.$; $p<0.0001)$; 21-29 months $\left.\left(F_{(1,94)}=73.7, p<0.0001\right)\right]$. We revealed a similar effect by examining for genotype differences of complete habituation abilities (i.e., "habituation difference") (see Materials and Methods) across all four trials by subtracting the normalized investigation duration during trial 4 from the maximum normalized investigation duration within that block (Fig. 1c). Indeed, even at our earliest time point, 3-4 months, the habituation index was 0.08 (i.e., $8 \%$ difference between WT and $\mathrm{Tg}$ mice in habituation abilities). This habituation deficit in $\mathrm{Tg}$ mice increased in 6- to 7 -month-old mice (31\% difference with
WT) and was maintained at both the 16- and 21- to 29-monthold age points (Fig. 1c)-demonstrating that atypical habituation to repeated odor exposure begins early in life in the Tg2576 mouse model and is maintained throughout life.

\section{Reduced odor discrimination in Tg2576 mice}

As a final behavioral measure, we assessed odor discrimination abilities across age in APP Tg mice. Odor cross-habituation-the response difference between a habituated odor and a newly presented odor - can be used as a test of spontaneous odor discrimination (Linster et al., 2002; Wesson et al., 2008), which eliminates the confounds associated with traditional operant odor discrimination tests (e.g., nutritional deprivation, sensorimotor control). A strong investigation of a novel odor after the presentation of a habituated odor (i.e., minimal crosshabituation) reflects discrimination of the two odors, whereas no or a weak investigation (i.e., strong cross-habituation) reflects generalization or a failure to discriminate (Wilson and Linster, 2008; Rankin et al., 2009). To our knowledge, no data are currently available on odor discrimination abilities in any line of $\mathrm{AD}$ model mice.

Therefore, to characterize odor discrimination abilities in Tg2576 mice, we analyzed the same odor cross-habituation data used for Figure 1, $b$ and $c$, but this time directly for odor crosshabituation. In particular, we compared across all trials, the difference in duration of investigating all novel odors (trial 1) from the previous trial 4. Because of differences in odor investigation in trial 1, normalized odor investigation data (as in Fig. 1c) were used. The investigation value for the preceding trial 4 was subtracted from the novel odor trial (trial 1) to calculate a "crosshabituation index." Similar measures of cross-habituation as an index for stimulus discrimination are commonly used across a variety of sensory paradigms (Rankin et al., 2009).

We found that Tg2576 mice showed reduced odor discrimination abilities (enhanced generalization) when compared with age-matched WT controls (Fig. 1d). Furthermore, similar to measures of odor habituation, deficits in odor discrimination were evident by 6-7 months of age. ANOVA on all crosshabituation values revealed a significant effect of genotype $\left(F_{(1,223)}=74.6 ; p<0.0001\right)$ on odor cross-habituation. There was a significant reduction in odor cross-habituation in 6- to 7 $\left(F_{(1,29)}=8.96 ; p=0.0056\right)-, 16\left(F_{(1,71)}=14.77 ; p=0.0003\right)$-, and 21 - to $29\left(F_{(1,81)}=222.0 ; p<0.0001\right)$-month-old Tg mice compared with controls (Fig. $1 d$ ). These results suggest that fine aspects of odor information processing in Tg2576 mice may be altered in a manner sufficient to yield deficiencies in odor discrimination and odor learning.

\section{Differential elevations in $\mathrm{A} \boldsymbol{\beta}$ throughout the olfactory system during aging}

Characterization of $\mathrm{A} \beta$ deposition throughout the olfactory processing stream in $\mathrm{AD}$ model mice, paired with perceptual data, would be valuable in understanding the pathological determinants of olfactory dysfunction in $\mathrm{AD}$. For instance, are $\mathrm{A} \beta$ levels related to olfactory dysfunction in a dose-dependent manner? Furthermore, given the differential expression of atypical olfactory behaviors throughout the life span reported here (Fig. 1), do increases in $\mathrm{A} \beta$ within certain olfactory regions temporally correspond with the emergence of these behaviors? Therefore, we characterized $A \beta$ levels throughout the olfactory system of mice previously tested in odor cross-habituation with two separate methods (see Materials and Methods)—Thioflavin-S labeling of $\beta$-pleated sheet confirmation of proteins, including fibrillar $\mathrm{A} \beta$ 


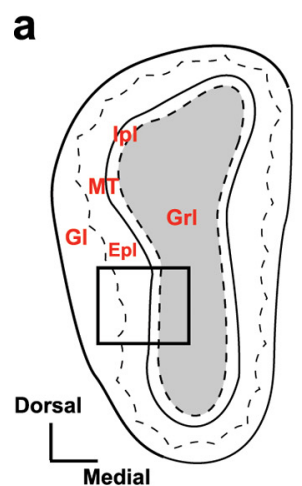

a

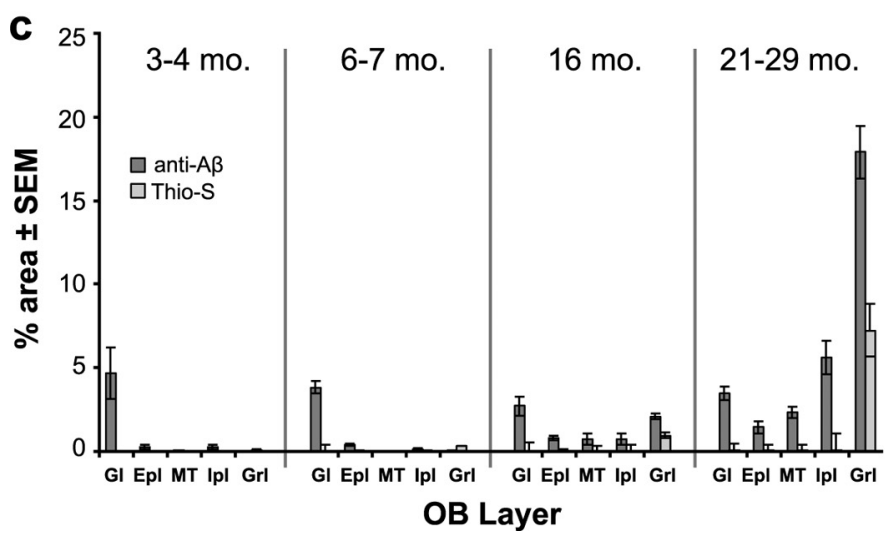

b 3-4 mo.

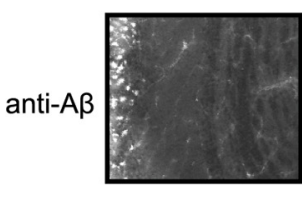

Thio-S
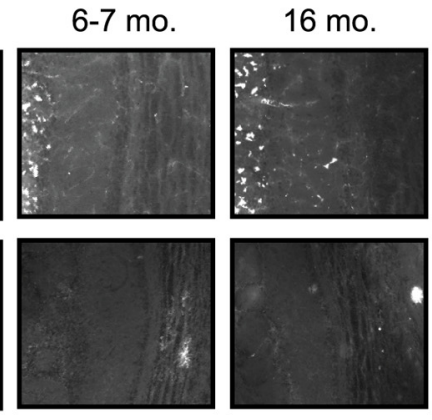

d

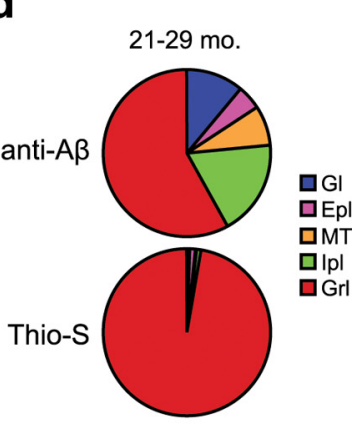

\section{1-29 mo.}

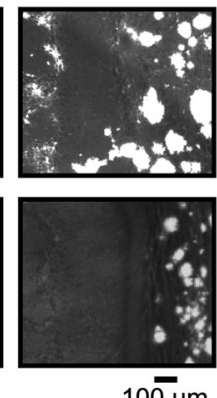

$100 \mu \mathrm{m}$

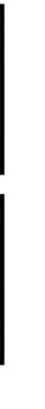


a
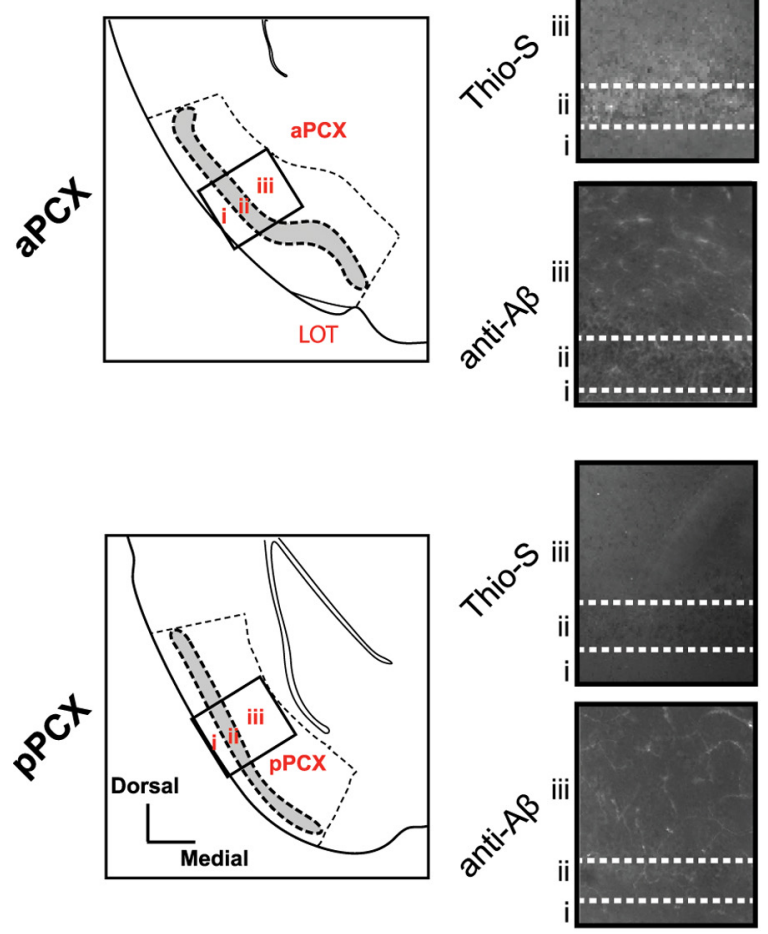

b

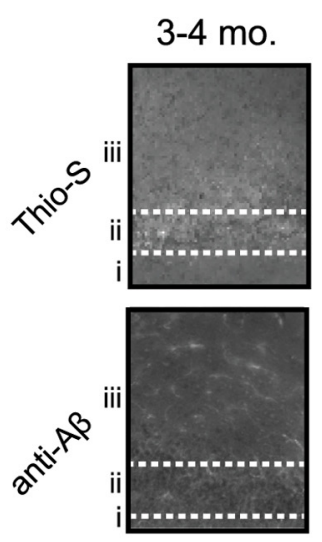

西
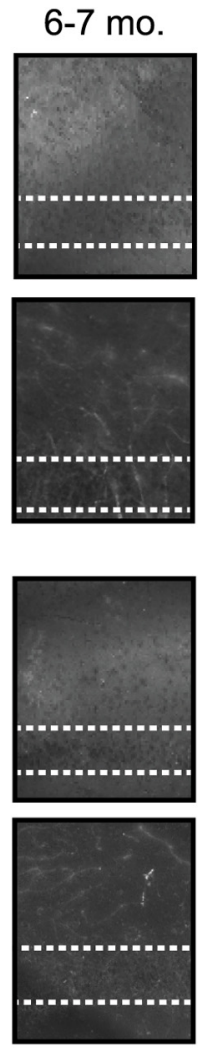
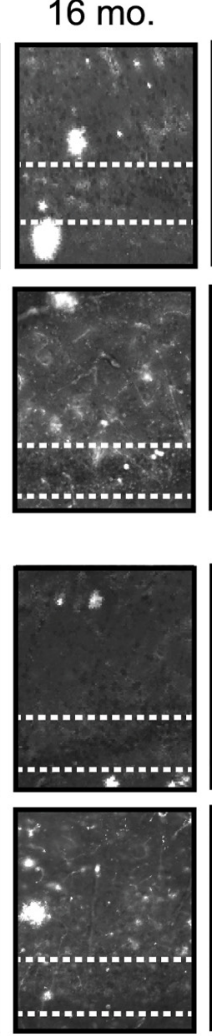

C
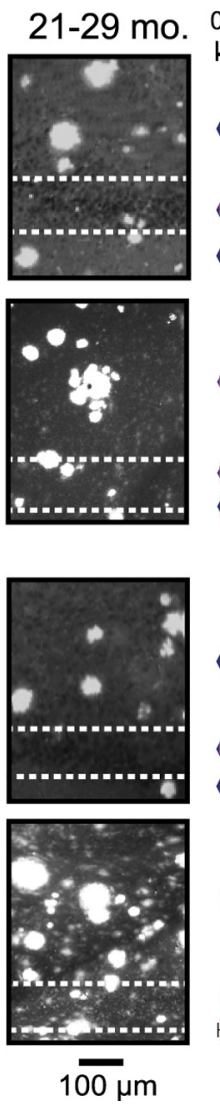

$\%$ area \pm SEM

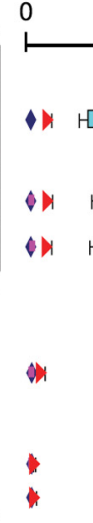

6

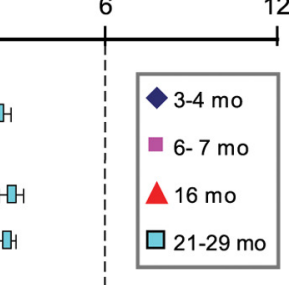

$1,0-1$

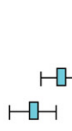

내

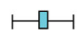

Figure 3. $A \beta$ deposits are distributed throughout $P C X$ cell layers. $\boldsymbol{a}$, Coronal plates of the $\mathrm{aPCX}$ (top) and pPCX (bottom). Diagrams show the locations of cell layers $\mathrm{i}$, ii, and iii. The dashed lines indicate regions used for quantification in $\boldsymbol{b}$. The black boxes indicate approximate locations of images used in $\boldsymbol{b}$. $\boldsymbol{b}$, Representative Thioflavin-S (Thio-S) and anti- $A \beta$ (4G8) staining in the aPCX (top) and PPCX (bottom) of Tg2576 mice at different age groups (mo, month). c, Quantification of A $\beta$ deposition (percentage area) within the PCX cortical layers (corresponding to arrangement in $\boldsymbol{b}$ ).

predict the occurrence of atypical odor habituation nor discrimination, as these behaviors emerged at 6-7 months of age. However, $\mathrm{A} \beta$ elevation at 16 months in the PCX did coincide with the emergence of atypical odor investigation behavior (PCXdependent short-term habituation) (Wilson and Linster, 2008) (Fig. 1b).

We also extended our analysis of $A \beta$ deposition into three "higher-order" olfactory regions. The OFC is located within the frontal cortex (Fig. 4a) and has reciprocal connections with the PCX (Illig, 2005). As shown in Figure $4 a$, we found $\mathrm{A} \beta$ deposition within the OFC starting with the 16 month age group. Furthermore, these $\mathrm{A} \beta$ levels increased into the 21-29 month age group, with the greatest escalation in anti- $\mathrm{A} \beta$-positive deposition (Fig. $4 a) . \mathrm{A} \beta$ deposits within the OFC were similar in density as in neighboring frontal cortex regions-suggesting that the OFC is not particularly affected by $\mathrm{A} \beta$ pathology as was the case for the $\mathrm{OB}$ Grl and layer III of the PCX. The Ent is located posterior to the pPCX (Fig. $4 b$ ) and receives small but direct MT cell input from the OB, as well as input from PCX (Schwob and Price, 1978). Furthermore, the Ent is involved in olfactory memory (Eichenbaum et al., 1994). We found relatively dense $A \beta$ deposition (both Thio-S- and anti-A $\beta$-positive) within the Ent of Tg2576 mice starting at the 16 month age group (Fig. 4b). As was the case for the OFC, regions neighboring the Ent displayed qualitatively similar levels of $\mathrm{A} \beta$ deposition. Finally, the Hipp is also involved in olfactory memory and receives olfactory-related input from the Ent (Stäubli et al., 1984) (Fig. 4c). Similar to previous reports (Hsiao et al., 1996), the Hipp was particularly affected by $\mathrm{A} \beta$ deposition (Fig. $4 c$ ). $\mathrm{A} \beta$ deposition was evident starting in the 16 month age group and progressed in levels into the 21-29 month group. Together, these results suggest that $\mathrm{A} \beta$ (deposition) pathology has the potential to impact olfactory processing across multiple brain areas.

To provide contrast to $\mathrm{A} \beta$ deposition levels within brain regions unique to olfactory processing, we also measured $\mathrm{A} \beta$ deposition within the primary somatosensory and motor cortices (cumulative percentage area in S1 and M1) (see Materials and Methods). Similar to that found in the PCX, OFC, Ent, and Hipp, $\mathrm{A} \beta$ deposition did not become evident in the S1/M1 until 16 months of age (percentage area anti- $\mathrm{A} \beta$ at 3-4 months: mean \pm SEM, $0.01 \pm 0.01 ; 6-7$ months: $0.1 \pm 0.03 ; 16$ months: $0.4 \pm 0.2$; and 21-29 months: $5.5 \pm 0.6$; percentage area Thio-S at 3-4 months: $0 \pm 0$; $6-7$ months: $0 \pm 0 ; 16$ months: $0.7 \pm 0.1$; and 21-29 months: $4.8 \pm 0.5$ ). Within animals, $\mathrm{A} \beta$ deposition was notably greater within principal olfactory areas (OB and PCX) than in S1/M1 (see Figs. 2, 3).

\section{Atypical olfactory behaviors indicate early-life elevations in $\mathrm{A} \boldsymbol{\beta}$ aggregation}

We observed $A \beta$ deposition across the majority of olfactory structures examined (Figs. 2-4). Therefore, we next asked whether Thio-S- and anti-A $\beta$-positive deposits correlate with the age-dependent olfactory behavioral deficits found in $\mathrm{Tg} 2576$ mice. To do this, we took the average percentage $A \beta$ values (Thioflavin S- and anti- $\mathrm{A} \beta$-positive separately, as shown in Figs. 2-4) from the OB, PCX, OFC, Ent, and Hipp within individual ani- 
a

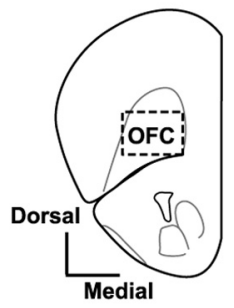

b

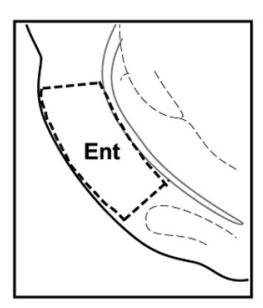

C

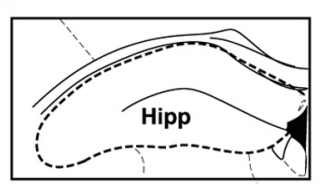

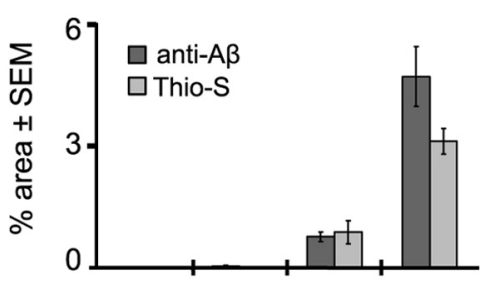
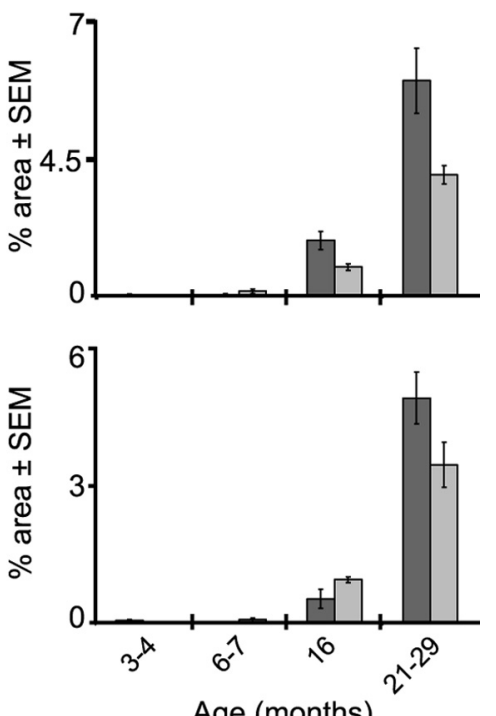

Age (months)

Figure 4. $A \beta$ deposition within higher-order olfactory regions. Coronal plate and quantification of $A \beta$ deposition [percentage area, Thioflavin-S (Thio-S) and anti-A $\beta$ (4G8)] in the OFC $(\boldsymbol{a})$, Ent $(\boldsymbol{b})$, and Hipp (c). The dashed regions within each coronal plate indicate approximate areas used for quantification. Error bars indicate SEM.

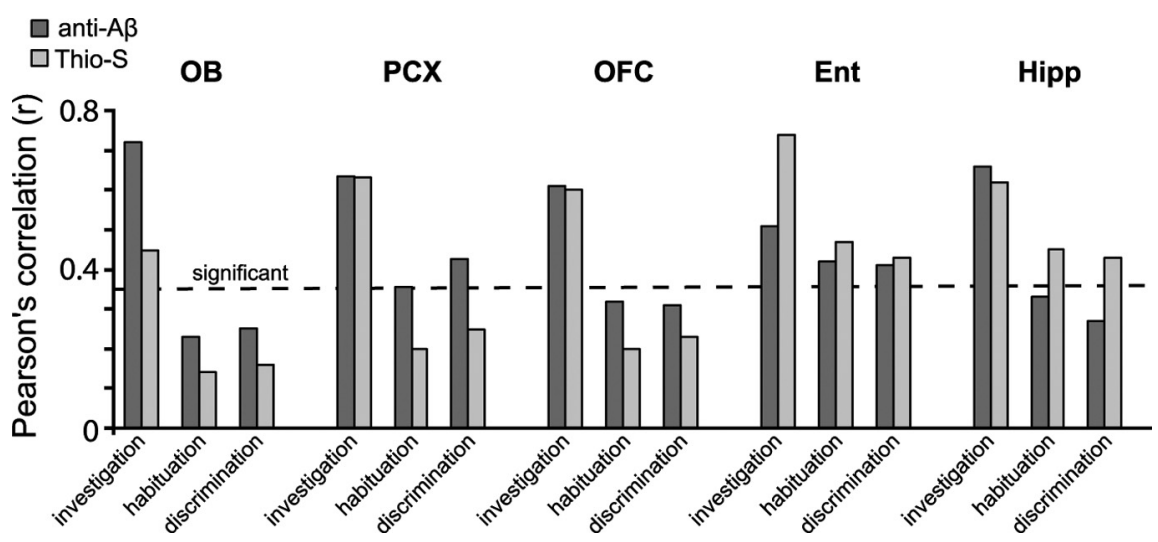

Figure 5. Olfactory behaviors correlate with the spatiotemporal deposition of $A \beta$. Pearson's correlation values ( $r$ ) between $A \beta$ deposition (percentage area) within the OB, PCX, OFC, Ent, and Hipp, and group-averaged olfactory scores (investigation time, percentage habituation, cross-habituation index) (Fig. 1c, black circles) in Tg2576 mice. Percentage habituation, The percentage difference between investigation duration in the first odor trial compared with the fourth. $O B$ data, Average of plaque area in granule cell layer. PCX data, Average of aPCX and pPCX, all cell layers. The dashed line indicates threshold of statistical significance $(r \geq 0.36)$ based on the population size within each bar ( $n=22$ mice).

mals (all age groups) previously tested on olfactory crosshabituation test (Fig. 1). As shown in Figure 5, behavioral deficits (investigation, habituation, and discrimination) significantly correlated $(r>0.37 ; n=22$ mice; $\mathrm{df}=20)$, in some cases highly, with both Thio-S- and anti-A $\beta$-positive $\mathrm{A} \beta$ burden. For example, abnormal odor investigation behaviors highly correlated with Thio-S-positive deposits in the Ent $(r=0.75)$ and with anti-A $\beta$ deposits in the $\mathrm{OB}(r=0.72)$. Odor discrimination abilities were significantly correlated with anti-A $\beta$ deposits in the PCX $(r=$ $0.75)$ and Ent $(r=0.43)$ and with Thio-S-positive deposits in the Hipp $(r=0.44)$, but with neither markers in the $\mathrm{OB}(r<0.25)$. Odor habituation abilities were significantly correlated with Thio-S-positive deposition in the Hipp $(r=0.46)$ and Ent $(r=$ $0.48)$ and with anti-A $\beta$ deposition in the Ent $(r=0.42)$ and PCX $(r=0.36)$ (Fig. 5). In contrast to previous reports suggesting that olfactory dysfunction is related to altered top-down modulation of odor processing (Guérin et al., 2009), our data revealing associations between $A \beta$ deposition and olfactory dysfunction support a potential link between $A \beta$ within specific areas of the primary olfactory network and some aspects of olfactory behavior (Fig. 6).

\section{Discussion}

The present results demonstrate that $\operatorname{Tg} 2576$ mice present with progressive olfactory impairments compared with age-matched WT controls. The Tg2576 mice behavioral deficits included (1) perseveration of initial odor investigation, (2) impaired shortterm odor habituation, and (3) impaired odor discrimination. Furthermore, detailed analysis of fibrillar (Thio-S positive) and fibrillar and/or nonfibrillar $\mathrm{A} \beta$ burden (anti- $\mathrm{A} \beta$ positive) throughout major olfactory processing centers revealed that the emergence of specific behavioral impairments corresponds with a progressive $\mathrm{A} \beta$ burden in specific olfactory regions. For example, although deficits in initial odor investigation strongly correlated with nonfibrillar $\mathrm{A} \beta$ deposits within the $\mathrm{OB}$, odor discrimination deficits were correlated with nonfibrillar $\mathrm{A} \beta$ deposits within PCX, but not OB. Finally, the earliest detectable olfactory deficits emerged as early as 3 months of age, the same age as emergence of initial nonfibrillar $A \beta$ deposits within the first synaptic layer of the OB. Together, these findings provide both a spatial and temporal correlation between specific olfactory deficits and localize $\mathrm{A} \beta$ neuropathology within the olfactory system (Fig. 6).

Tg2576 mice express an APP mutation (KM670/671NL), possess elevated soluble $\mathrm{A} \beta$ in the neocortex at 7 months of age (Kawarabayashi et al., 2001), and start displaying fibrillar $A \beta$ deposits in the hippocampus and neocortex $\sim 10$ months of age (Hsiao et al., 1996; Kawarabayashi et al., 2001). A $\beta$ plaques have been reported in the OBs of aged Tg2576 mice (Lehman et al., 2003) and humans with $\mathrm{AD}$ (Kovács, 2004). However, no detailed characterization of $\mathrm{A} \beta$ deposition within the $\mathrm{OB}$ nor throughout the olfactory system has been performed in either humans or AD model mice. Our analysis of $A \beta$ throughout the Tg2576 mouse olfactory system revealed differences both within and between olfactory structures in $\mathrm{A} \beta$ deposition. Although several studies to date have demonstrated that APP and/or A $\beta$ serve to modulate synaptic processing of neural activity (Kamenetz et al., 2003; Puzzo et al., 2008), whether $A \beta$ or the overproduction of other APP-processing fragments in these mice (e.g., C-terminal fragments) (Gao and Pimplikar, 2001) perturb network processing of information in the brain is currently not well understood. Indeed, measuring factors other than deposited $A \beta$ may be important in understanding the relationship between $\mathrm{AD}$ 


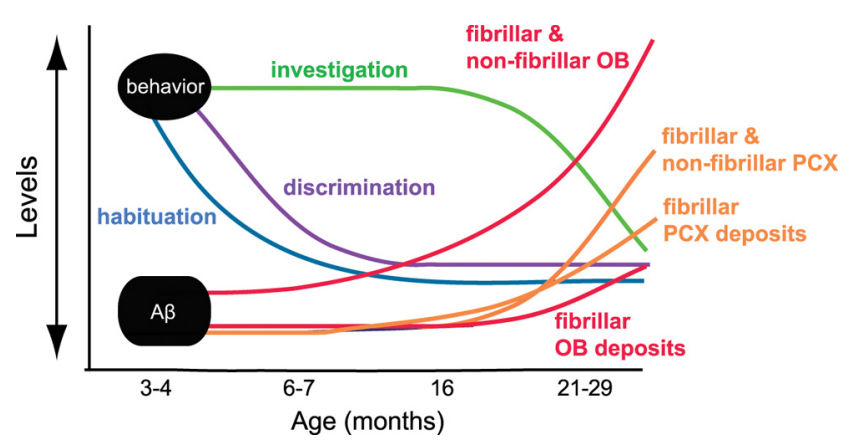

Figure 6. Summary diagram showing the emergence of atypical olfactory behaviors in relation to $A \beta$ deposition levels. $A \beta$ deposition [fibrillar (Thioflavin-S) and fibrillar and/or nonfibrillar (anti-A $\beta$ )] in the $O B$ (all layers) and $P C X$ (both anterior and posterior parts) in relation to olfactory behavioral performance throughout life in $\operatorname{Tg} 2576$ mice. Nonfibrillar $A \beta$ levels within the $O B$ were detectable at 3-4 months of age - on the start of impaired odor habituation. Atypical odor discrimination behaviors occur starting at 6-7 months of age. Abnormal odor investigation behavior did not begin until 16 months of age - before $O B A \beta$ escalation. Data (depicted in approximate "levels") are based on the results displayed in Figures 1-4.

pathology and the observed olfactory impairments. For example, soluble oligomeric forms of $\mathrm{A} \beta$ are important in mediating AD-like neurodegeneration (Varvel et al., 2008) and in modulating synaptic activity (Haass and Selkoe, 2007). Thus, although the present findings show strong relationships between both fibrillar and nonfibrillar $\mathrm{A} \beta$ deposits and atypical olfactory behaviors, they suggest that nonfibrillar forms of $\mathrm{A} \beta$ provide unique insights into the basis of olfactory dysfunction in AD. Furthermore, future studies into the potential contributions of other AD pathologies (e.g., NFTs) in combination with $\mathrm{A} \beta$ deposition to the emergence of $\mathrm{AD}$-related sensory loss may provide important insights into disease pathogenesis and allow additional understandings as to the cause of olfactory sensory loss in $\mathrm{AD}$.

The neural mechanisms that govern odor information processing in mammals are fairly well understood (Wilson and Stevenson, 2006). Such mechanisms are essential in informing perception and guiding olfactory behaviors. In the present study, we used the odor cross-habituation task, which takes advantage of the natural tendencies of mice to investigate novel odors (Sundberg et al., 1982). This test is less labor-intensive than the Morris water maze and both less invasive and less labor-intensive than fear-learning paradigms. The design of the cross-habituation task allowed us to assay three separate aspects of olfaction in the Tg2576 mouse, including novel odor-orienting responses, shortterm odor memory (habituation), and odor discrimination. This test will be highly advantageous in testing the efficacy of treatment against APP and/or A $\beta$-related abnormalities in AD mouse models.

On the detection of a novel odor in their environment, rats and mice reliably display an arousal-related orienting response involving sniffing and whisker movements in the direction of the odor. Such orienting responses are mostly used for the acquisition of odor information (Wachowiak, 2009). Indeed, a single sniff of an odor is sufficient to yield even complex odor detection and discrimination (Wesson et al., 2008)—arguing that prolonged rapid frequency sniffing (as seen in orienting responses) is not necessary to discriminate odors. Instead, the duration of this odor investigation behavior is dependent on arousal, motivation, and past experience (Wachowiak, 2009). Short-term habituation (decrease over repeated presentations) (Rankin et al., 2009) to odors is associated with receptor neuron adaptation (Reisert and Matthews, 2001; Verhagen et al., 2007), OB mitral cell adaptation (Kadohisa and Wilson, 2006a), and PCX adaptation (Wilson, 1998; Kadohisa and Wilson, 2006a). Despite these widespread factors, the primary contributor to short-term habituation is PCX adaptation, mediated by depression of mitral-pyramidal cell synapses within layer I of the PCX (Best and Wilson, 2004).

In particular, it is worth noting that PCX layer I, especially within the pPCX, had a high level of $\mathrm{A} \beta$ deposition. Given the importance of layer I synapses in short-term odor habituation, it is possible that the deficits in short-term odor habituation observed in Tg2576 mice were related to $\mathrm{A} \beta$-mediated mechanisms directly within layer I sensory processing. This hypothesis is supported by the fact that $\mathrm{A} \beta$ deposition in the PCX was apparent in 16-month-old mice, but not in 6- to 7-month-old mice-corresponding with the emergence in atypical short-term odor habituation (trial 1 odor investigation). Furthermore, anti-A $\beta$ staining was prevalent within the OB Gl layer starting at 3 months of age, which corresponds with the emergence of atypical odor habituation. The Gl is a crucial site for the initial processing of odor input from receptor neurons in the nasal cavity (Wachowiak and Shipley, 2006). Thus, it is possible that nonfibrillar $\mathrm{A} \beta$ acts directly on sensory neuron terminals within the Gl to modulate synaptic processing of odor information at the earliest level. Future studies will be important to examine these hypotheses.

Odor discrimination relies on olfactory receptor neuron function (Wachowiak and Shipley, 2006), local circuit function within the olfactory bulb (Beshel et al., 2007; Doucette et al., 2007), and pattern recognition and template formation of previously experienced odors within the PCX (Barnes et al., 2008). OB local circuit function, including granule cell-mediated feedback and lateral inhibition, is believed to contribute to contrast enhancement between similar odors (Wachowiak and Shipley, 2006; Wilson and Mainen, 2006). Notably, previous work has shown that the cross-habituation assay is sensitive to even slight differences in odor structure (Fletcher and Wilson, 2002). Association fiber plasticity within PCX layers I and III are believed to be critical for recognition and discrimination of those odorevoked spatiotemporal patterns of OB activity (Haberly, 2001). Thus, $\mathrm{A} \beta$ burden in either the $\mathrm{OB}$ or PCX could contribute to the observed odor discrimination deficits. As atypical odor discrimination emerged before $\mathrm{A} \beta$ deposition in the PCX, it is possible that the sparse $A \beta$ deposition within the OB Gl (as in Fig. 2) is responsible for the deficits in odor discrimination. In support of this, here, we found that the 6- to 7-month-old Tg2576 mice that had $\mathrm{A} \beta$ deposits in the $\mathrm{OB}$ had more predominant olfactory dysfunction than the mice that did not (as reflected through the correlations) (Fig. 5). Alternatively, maladaptive olfactory processing may arise within regions/layers neighboring these important layers (Liu et al., 2008). It will be important in future studies to determine the contributions of $\mathrm{A} \beta$ within each of these structures, independently, to the cellular processing of odor information.

In summary, our findings provide initial insights into the pathological basis of $\mathrm{AD}$-related olfactory dysfunction. In particular, our data revealed a correlation between the magnitude and occurrence olfactory deficits and the spatial-temporal pattern of $\mathrm{A} \beta$ deposition. Also, at 3 months of age, we observed nonfibrillar $\mathrm{A} \beta$ deposition within the olfactory bulbearlier than deposition within any other brain region. Therefore, these data suggest that nonfibrillar, versus fibrillar, 
$\mathrm{A} \beta$-related mechanisms likely contribute to early olfactory perceptual loss in Alzheimer's disease.

\section{References}

Albers MW, Tabert MH, Devanand DP (2006) Olfactory dysfunction as a predictor of neurodegenerative disease. Curr Neurol Neurosci Rep 6:379-386.

Alzheimer A, Stelzmann RA, Schnitzlein HN, Murtagh FR (1995) An English translation of Alzheimer's 1907 paper, "Uber eine eigenartige Erkankung der Hirnrinde." Clin Anat 8:429-431.

Barnes DC, Hofacer RD, Zaman AR, Rennaker RL, Wilson DA (2008) Olfactory perceptual stability and discrimination. Nat Neurosci 11:13781380.

Baum MJ, Keverne EB (2002) Sex difference in attraction thresholds for volatile odors from male and estrous female mouse urine. Horm Behav 41:213-219.

Beshel J, Kopell N, Kay LM (2007) Olfactory bulb gamma oscillations are enhanced with task demands. J Neurosci 27:8358-8365.

Best AR, Wilson DA (2004) Coordinate synaptic mechanisms contributing to olfactory cortical adaptation. J Neurosci 24:652-660.

Doucette W, Milder J, Restrepo D (2007) Adrenergic modulation of olfactory bulb circuitry affects odor discrimination. Learn Mem 14:539-547.

Eichenbaum HB, Otto T, Cohen NJ (1994) Two functional components of the hippocampal memory system. Behav Brain Sci 17:449-472.

Fletcher ML, Wilson DA (2002) Experience modifies olfactory acuity: acetylcholine-dependent learning decreases behavioral generalization between similar odorants. J Neurosci 22:RC201(1-5).

Gao Y, Pimplikar SW (2001) The $\gamma$-secretase-cleaved C-terminal fragment of amyloid precursor protein mediates signaling to the nucleus. Proc Natl Acad Sci U S A 98:14979-14984.

Gottfried JA, Winston JS, Dolan RJ (2006) Dissociable codes of odor quality and odorant structure in human piriform cortex. Neuron 49:467-479.

Guérin D, Sacquet J, Mandairon N, Jourdan F, Didier A (2009) Early locus coeruleus degeneration and olfactory dysfunctions in Tg2576 mice. Neurobiol Aging 30:272-283.

Haass C, Selkoe DJ (2007) Soluble protein oligomers in neurodegeneration: lessons from the Alzheimer's amyloid $\beta$-peptide. Nat Rev Mol Cell Biol 8:101-112.

Haberly LB (2001) Parallel-distributed processing in olfactory cortex: new insights from morphological and physiological analysis of neuronal circuitry. Chem Senses 26:551-576.

Hardy J, Selkoe DJ (2002) The amyloid hypothesis of Alzheimer's disease: progress and problems on the road to therapeutics. Science 297:353-356.

Hsiao K, Chapman P, Nilsen S, Eckman C, Harigaya Y, Younkin S, Yang F, Cole G (1996) Correlative memory deficits, Abeta elevation, and amyloid plaques in transgenic mice. Science 274:99-102.

Illig KR (2005) Projections from orbitofrontal cortex to anterior piriform cortex in the rat suggest a role in olfactory information processing. J Comp Neurol 488:224-231.

Kadohisa M, Wilson DA (2006a) Olfactory cortical adaptation facilitates detection of odors against background. J Neurophysiol 95:1888-1896.

Kadohisa M, Wilson DA (2006b) Separate encoding of identity and similarity of complex familiar odors in piriform cortex. Proc Natl Acad Sci U S A 103:15206-15211.

Kamenetz F, Tomita T, Hsieh H, Seabrook G, Borchelt D, Iwatsubo T, Sisodia S, Malinow R (2003) APP processing and synaptic function. Neuron 37:925-937.

Kawarabayashi T, Younkin LH, Saido TC, Shoji M, Ashe KH, Younkin SG (2001) Age-dependent changes in brain, CSF, and plasma amyloid $\beta$ protein in the Tg2576 transgenic mouse model of Alzheimer's disease. J Neurosci 21:372-381.

Kovács T (2004) Mechanisms of olfactory dysfunction in aging and neurodegenerative disorders. Ageing Res Rev 3:215-232.

Lee VM, Goedert M, Trojanowski JQ (2001) Neurodegenerative tauopathies. Annu Rev Neurosci 24:1121-1159.

Lehman EJ, Kulname LS, Lamb BT (2003) Alterations in beta-amyloid production and deposition in brain regions of two transgenic models. Neurobiol Aging 24:645-653.
Linster C, Johnson BA, Morse A, Yue E, Leon M (2002) Spontaneous versus reinforced olfactory discriminations. J Neurosci 22:6842-6845.

Linster C, Menon AV, Singh CY, Wilson DA (2009) Odor-specific habituation arises from interaction of afferent synaptic adaptation and intrinsic synaptic potentiation in olfactory cortex. Learn Mem 16:452-459.

Liu Y, Yoo M-J, Savonenko A, Stirling W, Price DL, Borchelt DR, Mamounas L, Lyons WE, Blue ME, Lee MK (2008) Amyloid pathology is associated with progressive monoaminergic neurodegeneration in a transgenic mouse model of Alzheimer's disease. J Neurosci 28:1380513814 .

Mesholam RI, Moberg PJ, Mahr RN, Doty RL (1998) Olfaction in neurodegenerative disease: a meta-analysis of olfactory functioning in Alzheimer's and Parkinson's diseases. Arch Neurol 55:84-90.

Mi W, Pawlik M, Sastre M, Jung SS, Radvinsky DS, Klein AM, Sommer J, Schmidt SD, Nixon RA, Mathews PM, Levy E (2007) Cystatin C inhibits amyloid- $\beta$ deposition in Alzheimer's disease mouse models. Nat Genet 39:1440-1442.

Montgomery KS, Simmons RK, Edwards G 3rd, Nicolle MM, Gluck MA, Myers CE, Bizon JL (2009) Novel age-dependent learning deficits in a mouse model of Alzheimer's disease: implications for translational research. Neurobiol Aging. Advance online publication. Retrieved November 22, 2009. doi:10.1016/j.neurobiolaging.2009.08.003.

Murphy C (1999) Loss of olfactory function in dementing disease. Physiol Behav 66:177-182.

Paxinos G, Franklin K (2000) The mouse brain in stereotaxic coordinates, Ed 2. San Diego: Academic.

Price JL, Davis PB, Morris JC, White DL (1991) The distribution of tangles, plaques and related immunohistochemical markers in healthy aging and Alzheimer's disease. Neurobiol Aging 12:295-312.

Puzzo D, Privitera L, Leznik E, Fà M, Staniszewski A, Palmeri A, Arancio O (2008) Picomolar amyloid- $\beta$ positively modulates synaptic plasticity and memory in hippocampus. J Neurosci 28:14537-14545.

Rankin CH, Abrams T, Barry RJ, Bhatnagar S, Clayton DF, Colombo J, Coppola G, Geyer MA, Glanzman DL, Marsland S, McSweeney FK, Wilson DA, Wu CF, Thompson RF (2009) Habituation revisited: An updated and revised description of the behavioral characteristics of habituation. Neurobiol Learn Mem 92:135-138.

Reisert J, Matthews HR (2001) Response properties of isolated mouse olfactory receptor cells. J Physiol 530:113-122.

Schwob JE, Price JL (1978) The cortical projection of the olfactory bulb: development in fetal and neonatal rats correlated with quantitative variations in adult rats. Brain Res 151:369-374.

Selkoe DJ (2008) Soluble oligomers of the amyloid $\beta$-protein impair synaptic plasticity and behavior. Behav Brain Res 192:106-113.

Stäubli U, Ivy G, Lynch G (1984) Hippocampal denervation causes rapid forgetting of olfactory information in rats. Proc Natl Acad Sci U S A 81:5885-5887.

Sundberg H, Døving K, Novikov S, Ursin H (1982) A method for studying responses and habituation to odors in rats. Behav Neural Biol 34:113-119.

Van Dijck A, Vloeberghs E, Van Dam D, Staufenbiel M, De Deyn PP (2008) Evaluation of the APP23-model for Alzheimer's disease in the odour paired-associate test for hippocampus-dependent memory. Behav Brain Res 190:147-151.

Varvel NH, Bhaskar K, Patil AR, Pimplikar SW, Herrup K, Lamb BT (2008) $\mathrm{A} \beta$ oligomers induce neuronal cell cycle events in Alzheimer's disease. J Neurosci 28:10786-10793.

Verhagen JV, Wesson DW, Netoff TI, White JA, Wachowiak M (2007) Sniffing controls an adaptive filter of sensory input to the olfactory bulb. Nat Neurosci 10:631-639.

Wachowiak M (2009) Active sensing in olfaction. In: The neurobiology of olfaction, Ed 1 (Simon SA, Nicolelis MA, eds), p 442. Boca Raton, FL: CRC.

Wachowiak M, Shipley MT (2006) Coding and synaptic processing of sensory information in the glomerular layer of the olfactory bulb. Semin Cell Dev Biol 17:411-423.

Wada E, Shigemoto R, Kinoshita A, Ohishi H, Mizuno N (1998) Metabotropic glutamate receptor subtypes in axon terminals of projection fibers from the main and accessory olfactory bulbs: a light and 
electron microscopic immunohistochemical study in the rat. J Comp Neurol 393:493-504.

Wesson DW, Carey RM, Verhagen JV, Wachowiak M (2008) Rapid encoding and perception of novel odors in the rat. PLoS Biol 6:e82.

Wilson DA (1998) Habituation of odor responses in the rat anterior piriform cortex. J Neurophysiol 79:1425-1440.

Wilson DA, Linster C (2008) Neurobiology of a simple memory. J Neurophysiol 100:2-7.

Wilson DA, Stevenson RJ (2006) Learning to smell: olfactory perception from neurobiology to behavior. Baltimore: The Johns Hopkins UP.

Wilson DA, Kadohisa M, Fletcher ML (2006) Cortical contributions to olfaction: plasticity and perception. Semin Cell Dev Biol 17:462-470.
Wilson RI, Mainen ZF (2006) Early events in olfactory processing. Annu Rev Neurosci 29:163-201.

Yadon CA, Wilson DA (2005) The role of metabotropic glutamate receptors and cortical adaptation in habituation of odor-guided behavior. Learn Mem 12:601-605.

Young JW, Sharkey J, Finlayson K (2009) Progressive impairment in olfactory working memory in a mouse model of mild cognitive impairment. Neurobiol Aging 30:1430-1443.

Zhuo JM, Prakasam A, Murray ME, Zhang HY, Baxter MG, Sambamurti K, Nicolle MM (2008) An increase in Abeta42 in the prefrontal cortex is associated with a reversal-learning impairment in Alzheimer's disease model Tg2576 APPsw mice. Curr Alzheimer Res 5:385-391. 